\title{
Therapeutic efficacy of mefloquine and sulfadoxine/ pyrimethamine for the treatment of uncomplicated pasmodium falciparum malaria in children, Metehara town, southeast Ethiopia
}

\author{
Moges Kassa ${ }^{1}$, Yared Mekonnen ${ }^{1}$, Tilahun Wolde Micheal ${ }^{1}$, Hussien Mohamed ${ }^{1}$, Samson Bulcha ${ }^{2}$
}

\begin{abstract}
Background: The development of resistance by $P$. falciparum to most drugs used in prophylaxis and therapeutics underscores the need to investigate the sensitivity of local parasite isolates to the currently available standard antimalarial drugs.

Objective: To assess parasitological resistance and therapeutic efficacy of mefloquine (MQ) and sulfadoxine/pyrimethamine (SP) in children with uncomplicated falciparum malaria in Metehara town, southeast Ethiopia.

Methods: The therapeutic responses to MQ and SP were examined using the World Health Organization 14-day in vivo test protocol. A total of 119 children that fulfilled the inclusion criteria were assigned to the MQ $(\mathrm{n}=59)$ or SP $(\mathrm{n}=50)$ treatment group. The patients were followed up for 14 days, and clinical and parasitological outcomes were assessed.

Results: The 14-day clinical and parasitological cure rates in children treated with MQ were found to be 100\% (55/ 55) with no recrudescence until day 14. In the SP group, the clinical cure and failure rates were found to be $78.9 \%$ $(45 / 57)$ and $12 \%$ (7/57) respectively. Out of the patients with clinical failure in the SP group, one child was classified as early treatment failure and six had late treatment failure. The incidence of parasitological resistance was $21.1 \%$ (8 patients with RI and, 4 patients with RII). MQ was faster in fever and parasite clearance rate by day $2(76.4 \%)$ and day $3(98.2 \%)$ than in the SP group (64.9\% day 2 and $91.2 \%$ day 3). Gametocyte carrier rate following therapy was significantly lower in those treated with MQ than in those receiving SP; $1.8 \%$ with MQ had gametocytes by day 14 compared to $50.9 \%$ with SP $(\mathrm{P}=<0.001)$. Adverse events to both drugs were mild and a few with no incidence of characteristics of MQ and SP induced psychosis or Steven Johnson, respectively.

Conclusion: These findings show that MQ $(15 \mathrm{mg}$ base $/ \mathrm{kg})$, administered as a single oral dose, was highly effective and well tolerated in the treatment of uncomplicated falciparum malaria achieving a cure rate of $100 \%$ of treated children in this endemic area. However, a dramatic increase in the frequency of parasite resistance to SP was demonstrated compared with the incidence two years previously. [Ethiop.J.Health Dev. 2005;19(3)167-173]
\end{abstract}

\section{Introduction}

Appropriate treatment with effective antimalarial drugs is accepted as a principal strategy for malaria control worldwide (1). The increasing resistance of Plasmodium falciparum to chloroquine (CQ), the mainstay of antimalarial therapy for the past 50 years, has made the choice of antimalarial therapy for prevention and treatment of this infection much more difficult in Africa (2). Widespread P. falciparum persistance to CQ has been associated with increased childhood mortality and morbidity in Africa (3). This situation has led to an increased use of drugs like halofantrine, which are more expensive and may be even toxic (4).

Sulfadoxine/pyrimethamine (SP) is usually considered the cheapest alternative to $\mathrm{CQ}$ for the treatment of uncomplicated falciparum malaria in large parts of Africa. Consequently, SP has replaced CQ as a first line therapy for uncomplicated falciparum in several African countries including, Malawi, Tanzania, Kenya and Botswana (2).
However, clinical resistance to SP has developed rapidly in Tanzania (5) and Kenya (6) where SP has already replaced CQ as a first line drug. Besides, strains of in vivo resistant $P$. falciparum have been reported in virtually all antimalarial drugs except artimisinin and its derivatives (7). Thus, such spread of resistance of $P$. falciparum to almost all drugs used in prophylaxis and therapeutics emphasizes the need for more information on the sensitivity of local parasite isolates to the available antimalarial drugs. Such information should lead national malaria control programs to adopt their therapeutic schedules to changes in $P$. falciparum susceptibility.

In Ethiopia, increasing CQ resistance has led to a national antimalarial policy change in 1999 from CQ to adopting $\mathrm{SP}$ as a first line drug for the treatment of uncomplicated falciparum malaria $(8,9)$. However, CQ still remains first line for $P$. vivax and $P$. malarae, with quinine being second line and reserved for severe malaria. An initial national survey undertaken between 1996 and 1998 by

${ }^{1}$ Ethiopian Health and Nutrition Research Institute (EHNRI); ${ }^{2}$ Metehara Sugar Estate Hospital, Central Etiopia

Email: Mogesk@hotmail.com, Tele. 766419, P.O. Box 1242, Addis Ababa, Ethiopia 
the $\mathrm{MOH}$ demonstrated less than $10 \%$ treatment failure for SP $(7,8)$. Since then, mean total treatment failure rates of $22-72 \%$ were reported from sentinel sites in $2003(10)$.

In Ethiopia, malaria patients infected with parasites that are resistant to SP or CQ are often treated with quinines, (9). However, quinines are largely unavailable at the lower level health facilities that operate around endemic areas. In view of the prevailing CQ resistant strains of $P$. falciparum, CQ of $300 \mathrm{mg}$ (as base, two tablets) weekly plus proguanil $200 \mathrm{mg}$ (two tablets) daily is currently recommended as a prophylaxis in the country (9). However, compliance with this regimen may be poor, due to the daily administration of proguanil. Mefloquine (MQ) is now widely recommended for antimalarial chemoprophylaxis worldwide $(11,12)$ and has been shown to be highly effective in the treatment of CQ and SPresistant $P$. falciparum in African children $(13,14)$ and pregnant women (15).

MQ has not yet been officially used for malaria control in Ethiopia. However, travellers coming to the country often take MQ as a prophylaxis. Currently, the Ministry of Health is also considering to introduce MQ for prophylaxis in the country (10). Despite its wide prophylactic use, MQ prophylaxis and treatment failure have been reported in some African countries even before the drug was widely used $(16,17)$. However, the authors of this study have not seen any published report of either in vivo or in vitro response of local malaria parasite isolates to $\mathrm{MQ}$.

In the event that MQ is to be added in the list of drugs for the treatment or prophylaxis of malaria in this country in the near future, a study was undertaken in 2002 that examined the in vivo sensitivity of $P$. falciparum parasites to MQ and SP in Metehara town. Comparison of the present SP sensitivity of $P$. falciparum was also made with results from previous studies conducted by the same team on the same site (18).

\section{Methods}

\section{Study area and population}

The study was carried out between October and November, 2002 during the peak transmission season at the outpatient department of Metehara Sugar Estate Hospital. Metehara is situated in the Rift Valley area, about $210 \mathrm{kms}$ east of Addis Ababa. The Estate irrigation system depends on the nearby Awash River for the cultivation of sugar-cane. The irrigation development, in turn, provides breeding sites for the Anopheles mosquito. Malaria transmission in this area occurs perennially with peaks during the two rainy seasons (September - November and March - May). This area is endemic for both $P$. falciparum and $P$. vivax, $P$. falciparum accounting for $80 \%$ of malarial infections while the remaining $20 \%$ is caused by $P$. vivax (Metehara Sugar Estate Hospital health records, unpublished data).
A previous trial at this study site demonstrated an $8 \%$ incidence of $P$. falciparum resistance to SP (18).

The study was based on the standard 14-day in vivo WHO protocol (19) except that the study population consisted of children aged one to seven years old who were attending the outpatient department with symptoms of malaria. WHO stresses the preferential inclusion of young children aged $<5$ years in all treatment efficacy studies even if all ages could be enrolled in areas of low transmission. The inclusion of children aged one to seven years old in this study was mainly due to the prevailing difficulty of attaining the target sample size.

The children were selected for the study according to the following criteria: axillary temperature of $\geq 37.5$ and $<$ $39.5{ }^{\circ} \mathrm{C}$ at the time of visit or a recent history of fever; a body weight of $>5 \mathrm{~kg}$; $P$. falciparum mono-infection, with asexual parasitemia ranging between 1000 to $140,000 / \mu 1$.; no antimalarial drug administration in the preceding two weeks before presentation; residing in the trial center or the surrounding village within a $15 \mathrm{~km}$ radius; haemoglobin of $>5.0 \mathrm{gm} / \mathrm{dL}$. Patients were recruited based on written informed consent obtained from patients' parents/guardians.

A child was excluded from the study if danger signs or concomitant febrile illnesses that interfere with outcome classification emerged during the follow-up period. Ethical clearance was obtained from the Research and Ethical Committee of the Ethiopian Health and Nutrition Research Institute.

\section{Treatment and follow-up}

A total of 762 children with symptoms suggestive of malaria were screened between October and November 2002. Overall 119(16\%) consecutive children aged 1- 7 years of age and who met the inclusion criteria were readily assigned to receive either MQ $(n=59)$ (mephaquin mepha Ltd, Aesch-Basel, Switzerland, 15 base $/ \mathrm{kg}$ ) or SP $(\mathrm{n}=60)$ (MALAREICH, Smithkline Beechman, sulfadoxine $25 \mathrm{mg} / \mathrm{kg}+$ pyrimethamine $1.25 \mathrm{mg} / \mathrm{kg}$ ) tablets on D0 (19). Tablets were given intact to older children or crushed in the form of syrup to young children. The dosage was adjusted according to the patient's body weight (7). Children with an axillary temperature of $38.5^{\circ} \mathrm{C}$ or higher were given a single dose of paracetamol tablet at $15 \mathrm{mg} / \mathrm{kg}$ and were treated with tepid sponging and fanning when necessary before administering the antimalarial drug. All children were given the medication orally under supervision and were monitored for $30 \mathrm{~min}$. If the children vomited the first dose, a second full dose was administered. Children vomiting more than once were withdrawn from the study immediately and admitted to the hospital for appropriate treatment. 
Children were followed at days 2, 3, 7 and 14. Parents were asked to bring the children to the hospital any time the children were sick. If the children failed to appear at the scheduled days, they were visited at home. On each follow-up visit, clinical examinations, temperature measurement, blood smears, presence of any adverse reactions to the drugs were recorded. Hemoglobin was measured at the day of registration and at day 14 with a colorimetric method using Drabkin's solution.

Thick and thin blood films were stained with Giemsa stains. All slides were read by a microscopist who did not have prior information as to the treatment groups and clinical outcomes. Parasite density was measured as the number of parasites per 200 leucocytes on a thick film, assuming total leucocytes of $8000 / \mu \mathrm{L}$. Blood films were considered negative if no asexual forms of $P$. falciparum were observed with 200 high power fields. Gametocytes were counted in thick blood smears against 1000 leukocytes and species confirmed on thin blood smears.

All treatment failures and dropout cases were treated in accordance with the National Malaria Treatment Policy Guideline (8).

\section{Outcome measures}

Outcomes were evaluated primarily by clinical and parasitological responses to therapy over 14 days (19). Clinical responses were defined as early treatment failure (ETF), if the patients developed signs of danger or severe malaria on or before day three, had fever and if axillary temperature was $\geq 37.5{ }^{\circ} \mathrm{C}$ on day two with parasitemia greater than the day zero count, or if axillary temperature was $\geq 37.5{ }^{0} \mathrm{C}$ on day three in the presence of parasitemia, or if parasitemia on day three was $\geq 25 \%$ of the count on day zero. Late treatment failure (LTF) means that parasitemia on day three was less than $25 \%$ of day zero count and development of signs of danger or severe malaria developed on any day from day four to day fourteen in the presence of parasitemia, or if parasitemia on day three was less than $25 \%$ of the count on day zero and axillary temperature was $\geq 37.5^{\circ} \mathrm{C}$ in the presence of parasitemia on any day from day four to day fourteen. All others were classified as adequate clinical response (ACR) cases. ACR means that parasitemia on day three was less than $25 \%$ of count on day zero and no parasitemia on day fourteen; or parasitemia on day three was less than $25 \%$ of the count on day zero even if parasites were present on day fourteen, and axillary temperature is below $37.5{ }^{\circ} \mathrm{C}$. Parasitological response was classified as S, RI, RII, RIII responses where, S indicates no parasite detectable on day fourteen; RI: day three parasite density $<25 \%$ of day zero density, no parasite detectable on day seven, and reappearance on day fourteen; RII: parasite density on day three $<25 \%$ of day zero density and parasites detectable on day seven and day fourteen; RIII: parasite detectable on day three and day seven (parasite count on day three $\geq 25 \%$ of initial parasite count on day zero). Secondary endpoints included fever and parasite clearance rates, presence of gametocytes on days, 3,7 , and 14, change in hemoglobin mean value from day zero to day fourteen and occurrence of adverse events. Adverse drug reaction is defined as the development of any symptom or sign that does not exist before the initiation of treatment and that is not a classic symptom of malaria.

Sample size: The sample size was calculated with an estimated 0.25 upper and 0.10 lower levels of treatment failure and with $80 \%$ power at a $5 \%$ significant level (19). Taking into account a dropout rate of $10 \%, 60$ patients per treatment group were enquired.

\section{Statistical analysis}

Data was fed into the SPSS computer software. A comparison was made between the two treatment groups using chi-square (for proportion) and t-tests (for normally distributed continuous data). Non-parametric tests, especially the Man-Whitney $U$ test, were employed as deemed appropriate.

\section{Results}

Out of $119(16 \%)$ children enrolled in the study, four children in the MQ group and three children in the SP group did not complete the 14 days follow up and were excluded from the study. The reasons for withdrawal included repeated vomiting following therapy (3 MQ, 1 SP), not available for follow ups (one in each group) and convulsion following therapy and were immediately admitted to the hospital. None of the enrolled children died. The overall dropout rate was $6 \%(7 / 119)$. Thus, 112 patients (55 MQ, 57 SP) have successfully completed the study and their data were evaluated.

The baseline demographic, clinical and laboratory data were compared between the two treatment groups (Table 1). There was, however, no significant difference between MQ and SP regimens with respect to the distribution of baseline attributes.

The14-day clinical and parasitological responses to therapy are shown in Tables 2. MQ was significantly more efficacious than SP in either clinical or parasitological responses. The clinical cure rate (ACR) for MQ was $100 \%$ compared with $87.7 \%$ for SP. There was no clinical or parasitological failure in the MQ group. In the SP group, the combined frequency of ETF and LTF was $21 \%$ (one child was classified as ETF and six-exhibited LTF).

Using the parasitological classification $21.1 \%(12 / 55)$ of the children in the SP group experienced RI/RII (8 RI, 4 RII) responses and $78.9 \%(45 / 47)$ showed the $S$ response. One patient who was classified as ETF on day three in the SP group had a parasite density lower than $25 \%$ of day zero and fitted the definition of RII. 
Table 1: Baseline characteristics of children who have completed the study and who were enrolled with uncomplicated Plasmodium falciparum and treated with mefloquine or sulfadoxine/pyrimethamine, Metehara, Ethiopia, Oct-Nov. 2002

\begin{tabular}{|c|c|c|c|}
\hline \multirow[b]{2}{*}{ Criteria } & \multicolumn{2}{|c|}{ Treatment group } & \multirow[b]{2}{*}{$P$ value } \\
\hline & $\begin{array}{l}\text { Mefloquine } \\
(n=55)\end{array}$ & $\begin{array}{l}\text { Sulfadoxine/pyrimethamine } \\
(n=57)\end{array}$ & \\
\hline $\operatorname{Age}^{a}$ (years) & $3.98(1.48)$ & $3.75(1.56)$ & 0.432 \\
\hline$<5$ years $(\%)$ & $38(69.1)$ & $43(75.4)$ & 0.528 \\
\hline Female(\%) & $27(49.1)$ & $26(45.6)$ & 0.850 \\
\hline Weight $^{\mathrm{a}}(\mathrm{kg})$ & $15.7(3.5)$ & $15.1(3.8)$ & 0.414 \\
\hline Temperature $^{a}\left({ }^{0} \mathrm{C}\right)$ & $38.5(0.92)$ & $38.4(0.82)$ & 0.242 \\
\hline No. $\geq 37.5$ & $54(98.2)$ & $50(87.7)$ & 0.726 \\
\hline Parasitaemia $^{\text {b }}($ per $\mu L)$ & $74773(1150-13955)$ & $6473(1000-13500)$ & 0.276 \\
\hline gametocyte rate(\%) & $4(7.3)$ & $7(12.3)$ & 0.528 \\
\hline Hemoglobine $\mathrm{g} / \mathrm{dL}^{\mathrm{a}}$ & $7.7(2.0)$ & $7.8(2.0)$ & 0.448 \\
\hline
\end{tabular}

${ }^{\mathrm{a}}$ Mean (SD) ${ }^{\mathrm{b}}$ Geometric mean (range)

The therapeutic responses of the infections to the drug treatments are summarized on Table 3 . The rate of fever clearance was significantly higher in children receiving MQ than those in the SP group. By days two and three, $87 \%$ and $96.3 \%$ of the children respectively in the MQ group were afebrile compared to $60 \%$ and $86 \%$ respectively, in the SP group. Most patients treated with MQ had their parasites cleared by day two (76.4\%) and day three $(98.2 \%)$ in the MQ group than in the SP group (64.9\% day 2 and $91.2 \%$ day 3 ). Nevertheless, these differences were not statistically significant. The gametocyte rate at baseline was not significantly different between the two treatments (7.3 MQ vs. 12.3 SP, $\mathrm{P}=0.53$ ) groups (Table 1). However, the proportions of patients with gametocyte on day $7(12.7 \%$ MQ vs. $66.1 \%$ SP,
$\mathrm{P}=<0.001)$ and day14 (1.8\% MQ vs. $50.9 \% \quad \mathrm{SP}$, $\mathrm{P}=<0.001$ ) were significantly lower in the MQ group than in the SP group (Table 3).

The mean haemoglobin level increased in the MQ group from an initial value of $7.7 \mathrm{~g} / \mathrm{dL}(\mathrm{SD}=2.0)$ at baseline (Table 1$)$ to $8.4 \mathrm{~g} / \mathrm{dL}(\mathrm{SD}=2.0))$ at day $14(\mathrm{P}=0.08)$ in children treated with MQ, but not in the SP group (7.8 vs. $7.8 \mathrm{~g} / \mathrm{dL}, \mathrm{P}=0.88$ ) (Table 3). Nevertheless, the difference in haematological responses between the two treatment groups was not statistically significant $(\mathrm{P}=0.11)$.

Adverse reactions to both drugs were mild and were resolved without treatment.

Table 2: Parasitological and clinical responses of children with uncomplicated falciparum malaria following treatment with mefloquine or sulfadoxine/pyrimethamine, Metehara, Ethiopia, Oct-Nov. 2002

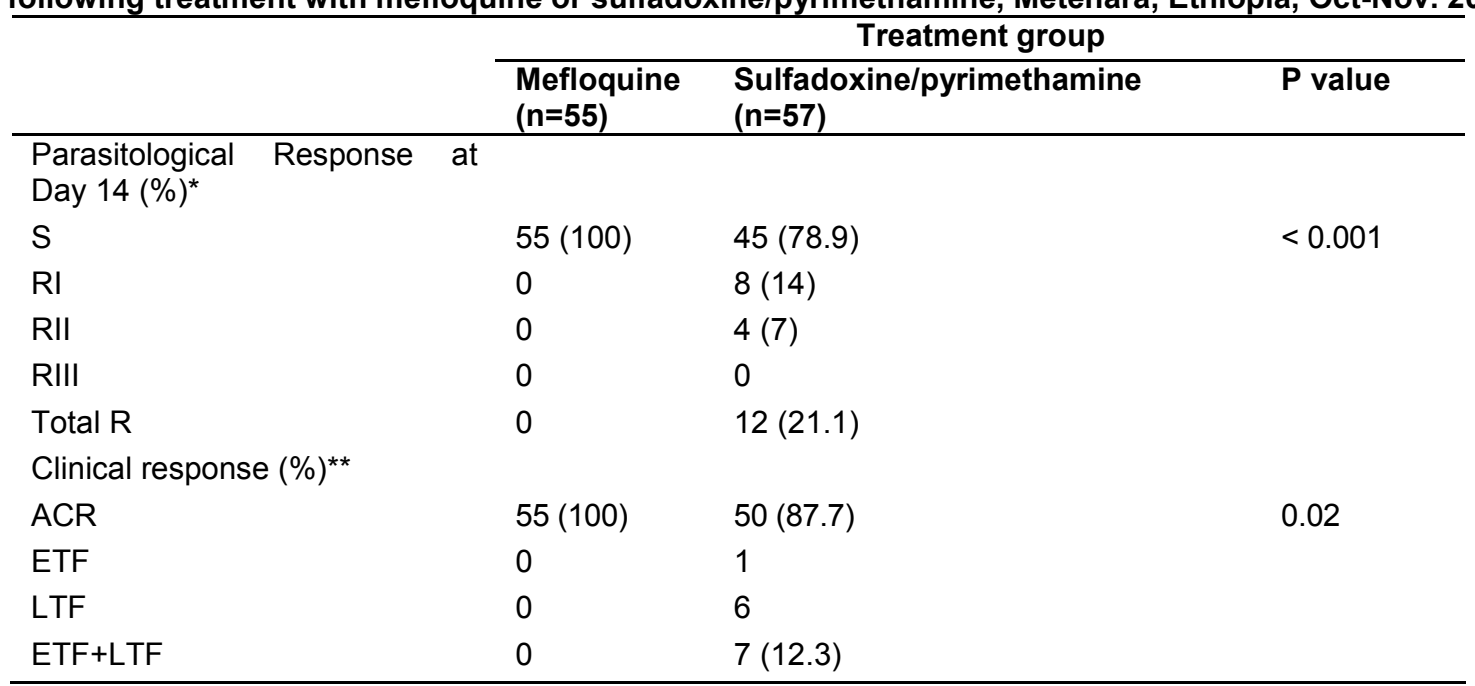

${ }^{\star} \mathrm{S}$, sensitive; $\mathrm{R}$ resistant

${ }^{* *} \mathrm{ACR}$, adequate clinical response; ETF, early treatment failure; LTF, late treatment failure.

See the text for details of the definitions of primary out come measures. 
Table 3: Therapeutic responses to mefloquine or sulfadoxine/pyrimethamine of children with uncomplicated falciparum malaria, Metehara, Ethiopia, Oct- Nov. 2002

\begin{tabular}{|c|c|c|c|}
\hline 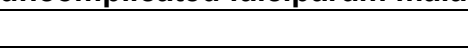 & 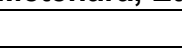 & Treatment Group & \\
\hline & $\begin{array}{l}\text { Mefloquine } \\
(\mathrm{n}=55)\end{array}$ & $\begin{array}{l}\text { Sulfadoxine/pyrimethamine } \\
(\mathrm{n}=57)\end{array}$ & P value \\
\hline \# fever cleared $(\%)^{++}$ & & & \\
\hline $\begin{array}{l}\text { Day } 2 \\
\text { Day } 3 \\
\text { \# parasite cleared (\%) }\end{array}$ & $\begin{array}{l}47(87.0) \\
52(96.3)\end{array}$ & $\begin{array}{l}30(60.0) \\
43(86.0)\end{array}$ & $\begin{array}{l}0.003 \\
0.010\end{array}$ \\
\hline $\begin{array}{c}\text { Day } 2 \\
\text { Day } 3 \\
\text { \# With gametocyte (\%) }\end{array}$ & $\begin{array}{l}42(76.4) \\
54(98.2)\end{array}$ & $\begin{array}{l}37(64.9) \\
52(91.2)\end{array}$ & $\begin{array}{l}0.217 \\
0.206\end{array}$ \\
\hline $\begin{array}{l}\text { Day } 3 \\
\text { Day } 7 \\
\text { Day } 14 \\
\text { Hemoglobin g/dL at Day14 (\%) }\end{array}$ & $\begin{array}{l}10(18.2) \\
7(12.7) \\
1(1.8) \\
8.3(2.0)\end{array}$ & $\begin{array}{l}7(12.3) \\
37(66.1) \\
28(50.9) \\
7.8(2.0)\end{array}$ & $\begin{array}{l}0.384 \\
<0.001 \\
<0.001 \\
0.114\end{array}$ \\
\hline
\end{tabular}

On admission, 5.1\% (3 of 59) of the children assigned to receive MQ vomited the medication twice compared with $1.7 \%$ (1 of 60$)$ of those in the SP group $(\mathrm{p}=0.59)$ and were withdrawn from the study. Vomiting occurred after drug administration in $7 \%$ (4 of 55) of the patients treated with MQ and $5.3 \%$ ( 3 of 57 ) of those treated with SP $(\mathrm{p}=0.96)$

Diarrhoea was reported in $16.4 \%$ (9 of 55) and $17.5 \%(10$ of 57) receiving MQ and $\mathrm{SP}(\mathrm{p}=0.93)$, respectively; $9.1 \%$
(5 of 55) of the children in the MQ and $15.8 \%$ (9 of 57) in the SP ( $\mathrm{p}=0.43)$ experienced reduced feeding; $3.6 \%$ (2of 55) children experienced abdominal pain in the MQ group and none in those given SP. Overall only one child experienced dizziness on days one and two following therapy with MQ, which was possibly considered to be related to treatment with MQ (Table 4).

Table 4: Adverse reactions in children with uncomplicated falciparum malaria following treatment with mefloquine or sulfadoxine/pyrimethamine, Metehara, Ethiopia ,Oct-Nov. 2002.

\begin{tabular}{llll}
\hline & & \multicolumn{1}{c}{ Treatment Group } & \\
& $\begin{array}{l}\text { Mefloquine } \mathbf{( n = 5 5 )} \\
\mathbf{n}(\%)\end{array}$ & $\begin{array}{l}\text { Sulfadoxine/pyrimethamine } \mathbf{( n = 5 7 )} \\
\mathbf{( \% )}\end{array}$ & P value \\
\hline Diarrhea & $9(16.4)$ & $10(17.5)$ & 0.93 \\
Reduced feeding & $5(9.1)$ & $9(15.8)$ & 0.43 \\
Vomiting & $4(7)$ & $3(5.3)$ & 0.96 \\
Abdominal pain & $2(3.6)$ & 0 & NA \\
Dizziness & 1 & 0 & NA \\
\hline
\end{tabular}

NA, not applicable

\section{Discussion}

The main finding of this trial was that MQ $(15 \mathrm{mg}$ base $/ \mathrm{kg}$ ) given as a single oral dose was highly effective and well tolerated in the treatment of uncomplicated falciparum malaria in children in this endemic area. All children who received MQ treatment were cured with no recrudescence until day 14 . These findings are consistent with results from previous studies that have shown a $100 \%$ cure rate with MQ in Nigerian children (13). However, these data contrast with findings in Cameron (16) and Malawi (17), where high failure rate of MQ was observed even before it was being widely used.

The result of this study also indicated a dramatic increase in the incidence of SP resistance in this area. SP resistance was $8 \%$ in 2000 (18) and this figure has risen to $21 \%$ in the present study. This represents a marked increase in resistance compared with the same area 2 years earlier. In support of the results of this study a more recent national survey undertaken by the Ministry of Health has revealed a total treatment failure rate of about $22 \%$ for SP at Metehara (10). Similarly, P. falciparum resistance to SP has reached severe levels in neighbouring East African countries that adopted SP as a first line drug $(4,5)$.

Both drugs are not gametocidal (7). However, in the present study treatment with SP was associated with a significantly higher level of gametocyte rate than treatment with MQ. More than $50 \%$ of patients treated 
with SP had gametocytes on day 14.These findings are consistent with results from previous studies in the same study area (18) and elsewhere (20), which showed a similar increased gametocyte rate following treatment with SP. Such increase in gametocyte carrier rate might escalate the transmission of $P$. falciparum and the spread of resistant parasites in the study area.

Adverse events to both drugs were mild and few. Besides, no clinically relevant difference between the study groups was observed with respect to adverse drug effects. Mefloquine has been associated with psychosis (21) however, such psychiatric reaction has never been observed with the use of MQ (15 mg base $/ \mathrm{kg}$ ) at the dose used for the treatment of malaria in this area. Vomiting and nausea were the most common MQ related adverse reactions (7). These occurred in only four and one child treated with this drug respectively. Besides, we recorded no incidence of Steven Johnson under SP treatment, a well-known side effect of this drug (7)

These findings show that MQ (15mg base $/ \mathrm{kg})$, administered as a single oral dose, was highly effective and well tolerated in the treatment of uncomplicated falciparum malaria, achieving a cure rate of $100 \%$ in treated children in Metehara. Given that MQ is effective against the blood forms of $P$. vivax malaria (7) it appears to be a valuable addition to the list of drugs for prophylaxis and the treatment of malaria in the country. Increasing SP resistance in P. falciparum in the study area is among the most important findings of this study. In view of the high level of SP resistance, the Ministry of Health is currently in the process of changing the first line drug from SP to Coartem a combination of artemether and lumefanthrine. However, the treatment is too expensive for wider use in resource poor countries such as Ethiopia. Although there is international support for Coartem at the moment, its sustainability in the long run is an area of concern. In addition, the diagnostic facilities should be strengthened to ensure the use of these valuable drugs only to laboratory confirmed cases.

The main limitation of this study is that it stopped at 14 days of follow up which might have been too early to judge the occurrence of recrudescence or late emergent parasites under MQ treatment. Previous studies have demonstrated that most failures with MQ occur after a month following therapy (22). These studies suggest follow-up periods up to 42 days to detect recrudescence parasites with MQ treatment. Therefore, the standard 14day follow up recommended by WHO (19) for areas of intense transmission, which is applied in the present study might not have been sufficient to detect the late emergence or resistance of parasites with MQ. In this context, our results may underestimate the true recrudescence rate. This however does not affect the study outcome very much given that the WHO standard test method is still not well suited to differentiate new infection from recrudescence if the length of follow up period is extended beyond 14 days (19).

\section{Acknowledgements}

We thank the Clinical Study Team of Metehara Hospital particularly to Ato Seyum Hagos and, Ato Kassa Hailu for their technical support. Our thanks also go to all participating children and their parents for their friendly participation in the study. We are also grateful to $\mathrm{Dr}$ Yigeremu Abebe for providing the mefloquine necessary for the study. This study was financially supported by EHNRI.

\section{References}

1. World Health Organization. Malaria. WHO, Geneva. WHO Technical Report Series, No.8928, 2000.

2. White NJ, Nosten F, Looareesuwan S. et al. Averting a malaria disaster. The Lancet, 1999;353:1965-7.

3. Trape JF, Pison G, Presiosi et al. Impact of chloroquine resistance on malaria mortality. Acadamie des Sciences Paris, Serie III, 1998;321:689-697.

4. Bloland P B. and Ettling M. Making malaria treatment policy in the face of drug resistance. Ann Trop Med Parasitol, 1999;93:5-23.

5. Mutabingwa T, Nzila AM, Mberu EK. et al. Chloroproguanil-dapson for treatment of drugresistant falciparum malaria in Tanzania. The Lancet, 2001;358(9289):1218-23.

6. Nzila A.M, Mberu E K, Sulo J. et al. Towards an understanding of the mechanism of pyrimethaminesulfadoxine resistance in Plasmodium falciparum: Genotyping of dihydrofolate reductase and dihydropteroate synthase of Kenyan parasites. Antimicrob Agents Chemother, 2000;44:991-6.

7. World Health Organization. The use of antimalarial drugs. Report of a WHO Technical Consultation. World Health Organization, Geneva, Switzerland 2001 (document WHO/CDS/RBM/2001.33).

8. MOH. Draft report on therapeutic efficacy of chloroquine, amodiaquine and sulfadoxinepyrimethamine for treatment of uncomplicated falciparum malaria in Ethiopia. Malaria and Other Vector Borne Diseases Control Unit. Epidemiology and AIDS Department MOH. 1998.

9. MOH Malaria and Other Vector Borne Diseases Control Unit Epidemiology and AIDS Department, Ministry of Health. Malaria diagnosis and treatment guidelines for health workers in Ethiopia, 1999, Addis Ababa Ethiopia.

10. MOH. Proceedings of the national workshop on antimalarial treatment policy in Ethiopia, 25-26 May 2004, Addis Ababa, Ethiopia.

11. World Health Organization. International travel and health. Vaccination requirements and health advice. WHO Office of Information, Geneva, Switzerland 1995. 
12. Centres for Disease Control. Health information for international travellers. US Department of Health and Human Services Atlanta, USA., 1994.

13. Sowunmi A and Oduola AMJ. Comparative efficacy of chloroquine/chloropheniramin combination and mefloquine for the treatment of chloroquine-resistant Plasmodium falciparum malaria in Nigerian children. Trans. Roy Soc Trop Med Hyg. 1997;91:689-693.

14. Makono R and Sibanda S. Review of the prevalence of malaria in Zimbabwe with specific reference to parasite drug resistance (1984-96). Trans Roy Soc Trop Med Hyg. 1999;93:449-452.

15. Steketee RW, Wirima J J, Slutsker L. et al. Malaria parasite infection during pregnancy and at delivery in mother placenta and new born: Efficacy of chloroquine and mefloquine in rural Malawi, Am J Trop Med Hyg. 1996, Supplement, 24-32.

16. Brasseur P, Kouamouo J, Moyou-Somo R. and Rruilhe P. Multi-drug resistant falciparum malaria in Cameroon in 1987-88. II. Mefloquine resistance confirmed in vivo and in vitro and its correlation with quinine resistance. Am J Trop Med Hyg. 1992;46:8-14.

17. MacArthur J, Stennies G, Macheso A, et al. Efficacy of mefloquine and sulfadoxine/pyrimethamine for the treatment of uncomplicated Plasmodium falciparum infection in Machinga district, Malawi, 1998. Am J Trop Med Hyg. 2001;65:679-684.
18. Kassa $\mathrm{M}$, Mengesha $\mathrm{T}$ Mekonnen $\mathrm{Y}$, Balcha $\mathrm{F}$, Mohammed $\mathrm{H}$. and Bulcha S. A randomized trial of sulfadoxine/pyrimethamine plus chloroquine versus sulfadoxine/pyrimethamine alone for the treatment of uncomplicated falciparum malaria in children, Metehara, Central Ethiopia. Eth.Pharm J, 2001; 22:16-9.

19. World Health Organization. Assessment of therapeutic efficacy of antimalarial drugs: for uncomplicated falciparum malaria in areas with intense transmission. World Health Organization, 1996 (document WHO/MAL/96.1077) Geneva, Switzerland.

20. Sowunmi A, Oduola AMJ, Ogundahunsi OAT and Salako LA. Comparative efficacy of chloroquine plus chlorpheniramine and pyrimethamine/sulfadoxine in acute uncomplicated falciparum malaria in Nigerian children, Trans Roy Soc Trop Med Hyg, 1998;92:7781.

21. Weinke $T$, Trautmann $M$, Held $T$, et al. Neurosychatric side effects after the use of mefloquine. AM J Trop Med Hyg. 1991;45:86-91.

22. Ter Kuile FO, Dolan G, Nosten $F$ et al. Halofanthrine versus mefloquine in treatment of multidrug resistant falciparum malaria. The Lancet, 1993;341:1044-1049. 
